\title{
Atrial fibrillation care improvement collaborative
}

\author{
Paul Robelia, Stephen Kopecky, Tom Thacher
}

Mayo Clinic Rochester, USA

\begin{abstract}
Atrial fibrillation (AF) is an increasingly common cardiac arrhythmia. Many patients with new onset or recurrent AF present to the emergency department and are subsequently admitted to the hospital and seen by cardiology specialists for follow up. In an attempt to address this high utilization of acute health care resources, reduce costs, and improve patient care, our institution instituted a collaborative project between the departments of emergency medicine, cardiology, family medicine, and primary care internal medicine.
\end{abstract}

The project team oversaw development of a new emergency department AF order set, encouraged utilization of a new oral anticoagulant (dabigatran), improved the primary care follow up connection, and deployed a multimodal education plan for primary care providers. Between 2012 and 2014, these interventions resulted in a 17\% reduction in total AF per member per month (PMPM) cost, a $28 \%$ reduction in AF PMPM inpatient cost, and a $24 \%$ reduction in inpatient admissions for $\mathrm{AF}$.

\section{Problem}

Total PMPM costs for the community primary care practice at Mayo Clinic Rochester (Minnesota, USA) were continuing to increase in 2012 (per member per month cost is the average cost of health care for a group of patients, and is calculated by dividing the cost of care by the number of patients and number of months). Significant cardiovascular resources were being spent on this group of patients, which impacted the ability to apply resources to other care initiatives. Specifically, emergency department (ED) and inpatient care utilization and costs for community primary care patients with atrial fibrillation were higher than desired. This is detrimental to patients and represents an ineffective allocation of system resources.

Drivers for inpatient admission of AF patients and subsequent cardiology follow up include nonstandardized ED care protocols, lack of ED provider awareness of primary care follow up capabilities, complexity of low molecular weight heparin and warfarin anticoagulation, and primary care provider unfamiliarity with AF care standards.

\section{Background}

Atrial fibrillation is the most common sustained cardiac arrhythmia, with a prevalence of $0.4-1 \%$ in the general population. Over the past 20 years, hospital admissions for AF have increased $66 \%$, accounting for approximately one-third of hospitalizations for cardiac rhythm disturbances.[1] AF is a costly public health problem (\$US 3600 per patient annually), with hospitalizations, drugs, and consultations being the primary cost drivers.[1]

Similarly, in 2012 total per member per month costs for local primary care patients at Mayo Clinic Rochester were increasing, and AF care represented $2 \%$ of these total costs. Significant cardiovascular resources were being spent on these patients; prior departmental efforts had reduced the hospital admission rate for AF from $75 \%$ to $51 \%$.

Previous studies showed that AF patients managed by their primary care providers received appropriate therapy, but high hospitalization rates persisted.[2] Other efforts have shown that shared care of congestive heart failure patients by primary care providers and specialists after hospital discharge can extend patient survival.[3] Integrated cardiology and primary care services can improve follow up and chronic treatment of patients with ischemic heart disease, heart failure, and atrial fibrillation while increasing primary care provider satisfaction and stabilizing resource utilization.[4] Novel care systems incorporating an observation unit and a short term outpatient follow up clinic have been deployed, significantly reducing hospital admission rates regardless of comorbidities.[5]

Low molecular weight heparin injections and warfarin have served as the cornerstones of therapeutic anticoagulation for atrial fibrillation in the past, but the complexity of this regimen can challenge patients and drive hospital admission. The new oral anticoagulants dabigatran, apixaban, and rivaroxaban are effective alternatives to warfarin for stroke prophylaxis in patients with nonvalvular atrial fibrillation.[6]

Approximately 650 patients are seen in the Mayo Clinic Rochester emergency department for AF yearly, and one-third of these are community primary care patients. Many of these patients, although stable, were seen in follow up by a cardiology specialist rather than their primary care provider. This potentially impacted continuity of care and development of long term care plans.

\section{Baseline measurement}

The project group identified key metrics for community primary care patients: per member per month (PMPM) total cardiovascular care cost, PMPM atrial fibrillation (AF) care costs, and inpatient care costs. Counterbalance measures were 30 day readmission rate and 
emergency department return rate. Data were obtained from Mayo Clinic financial accounting services.

- Total AF patients in the community primary care practice (2012): 4018

- Inpatient admissions for AF (2012): 299

- Emergency Department return visits for AF in 2012

(countermeasure): 142

- Thirty day readmissions for AF in 2012 (countermeasure): 58

- Additionally, visits to the outpatient clinic for AF were tracked: 2675 in 2012

\section{Design}

Key stakeholders from family medicine, primary care internal medicine, emergency medicine, and cardiology were identified and invited to participate in the project group. Subsequent interviews of stakeholders identified contributing causes, including variable primary care provider comfort with managing $A F$, unclear ED follow up plan, lack of standardized ED protocol for AF care, unclear long term AF care plan, complexity of warfarin management, and inconsistent access to primary care follow up. High level opportunities for improvement included standardization of ED care, identification, and utilization of an alternative anticoagulant (dabigatran), and optimization of primary care provider follow up.

This project took place at Mayo Clinic Rochester (Minnesota, USA). It involved collaboration between nursing, physician, and desk staff from the departments of family medicine, primary care internal medicine, cardiology, and the emergency department. An institutional charter was written and approved to ensure high level leadership support.

\section{Strategy}

The project team began meeting in February 2013. A standardized atrial fibrillation order set was developed for the emergency department, addressing the preferred rate control medication (diltiazem) and an alternative anticoagulant (dabigatran). The calcium channel blocker diltiazem was chosen rather than a beta blocker (such as esmolol) for several reasons including literature support for its use,[7] the ability to use in patients with bronchospastic airway disease, the ability to administer as an IV drip via nomogram, provider familiarity, and ability to easily convert to oral therapy.

Dabigatran, rather than the more complex low molecular weight heparin and warfarin regimen, was offered as an option to patients when appropriate. Dabigatran was the only novel oral anticoagulant (NOAC) available for atrial fibrillation stroke prophylaxis when project planning began, and the project group elected to stay with a single agent through implementation despite availability of other NOACs subsequently. A 30 day supply of dabigatran was provided to patients who chose this option.

The emergency department atrial fibrillation order set also encompassed stroke risk calculation using the CHADS2 score, the role and timing of cardioversion, and utilization of the emergency department observation unit.

Stable local AF patients followed up with their primary care providers, rather than cardiologists, whenever possible. Cardiology and emergency department project champions communicated the order set and practice changes to their providers. Primary care project champions educated family medicine and primary care internal medicine staff via email and intranet, face to face presentations, and educational videos. Project leadership participated in an institution wide panel presentation and question and answer session. Initial rollout took place in July 2013.

The project group continued to meet after rollout to troubleshoot several issues, including the process of developing an AF order set for a busy emergency department. This task was completed within the project timeline. Additionally, providing a 30 day supply of dabigatran to patients proved challenging, but a medication discount applied within the pharmacy system improved access to this medication. Finally, the problematic connections between the emergency department control desk and the primary care practice scheduling desks were improved by providing the ED with phone numbers, electronic message access and clinic hours while at the same time educating primary care schedulers regarding prioritization of follow up for $\mathrm{AF}$ patients.

\section{Results}

The project group monitored key metrics and countermeasures throughout implementation with the assistance of Mayo Clinic financial accounting services. Project outcomes in 2013 and 2014 included:

- Total atrial fibrillation (AF) patients in the community primary care practice (2014): 4,093 (1.9\% increase from 2012)

- Inpatient admissions for AF: 299 in 2012, 248 in 2013 (-17\%), and 228 in $2014(-8 \% 2013$ to 2014$)$

- Clinic outpatient visits for AF: 2675 in 2012, 2662 in 2013

$(-0.48 \%)$, and 2870 in $2014(+7.8 \% 2013$ to 2014$)$

- Total per member per month (PMPM) cost for AF care in the community primary care practice: $-12.2 \% 2012$ to $2013,-4.9 \% 2013$ to 2014

- Total PMPM cost for inpatient AF care in the community primary care practice: $-20.6 \% 2012$ to $2013,-7.5 \% 2013$ to 2014

- Emergency Department return visits for AF (countermeasure): 142 in 2012, 132 in 2013 (-7\%), and 119 in 2014 (-9.8\% 2013 to 2014)

- Thirty day readmissions for AF (countermeasure): 58 in 2012, 49 in $2013(-16 \%)$, and 42 in $2014(-14 \% 2013$ to 2014). 
The attached run chart demonstrates reduced AF inpatient admissions in the face of stable emergency department visits and 30 day readmission rates for $\mathrm{AF}$ across the project implementation timeline.

An eight week audit of community practice AF patients seen in the emergency department performed one year post implementation (July and August 2014) showed two of 42 patients (4.8\%) already on dabigatran and four of $42(9.5 \%)$ started on dabigatran. All of these patients were discharged from the emergency department rather than admitted to the hospital.

These results demonstrate sustained reduction in care costs for community AF patients and reflect benefit to AF patients through reduced utilization of acute healthcare resources. Identified countermeasures (emergency department AF return visits and 30 day $A F$ readmission rates) were stable, indicating no adverse effect on $\mathrm{AF}$ patient care.

See supplementary file: ds5932.pptx - "Atrial Fibrillation Collaborative Project Run Chart and Outcomes Charts"

\section{Lessons and limitations}

Project leaders encountered several challenges during implementation, including the task of educating large groups of providers from several departments (cardiology, emergency medicine, primary care internal medicine, and family medicine) practicing at multiple sites. Emails, intranet videos, web pages, face to face presentations, and appropriate reinforcement by practice leadership were utilized to disseminate project education.

Success of this project largely depended on increasing scheduled follow up with primary care providers after emergency department visits. The project group found it difficult to bring the geographically separate and functionally distinct ED and primary care clinic schedulers together for this purpose. Representatives from primary care reviewed patient cases early in implementation and facilitated improved communication between these two groups.

Another key project innovation involved availability of the alternate anticoagulant dabigatran. The logistics of providing a 30 day supply of this costly medication to patients who chose this option in the ED proved challenging, but a medication discount provided within the pharmacy system addressed this issue successfully.

Interdepartmental collaboration in a large healthcare institution presents multiple challenges including divergent priorities and resource allocation. Identifying shared priorities (improving patient care and reducing cost) and addressing concerns (follow up planning) immediately were important breakthroughs for the group.

\section{Conclusion}

Interdepartmental collaboration and care standardization offer opportunities to improve quality of care and reduce costs for patients with atrial fibrillation. These patients are able to avoid inpatient hospitalization, benefit from a new anticoagulation option, and follow up more closely with their primary care providers in order to address long term care plans. Engagement of all members of the care team allowed the project group to develop a novel approach that provides these patients improved care at lower cost.

\section{References}

1. Fuster V, Ryden LE, Cannom DS, Crijns HJ, Curtis AB, Ellenbogen KA, et al. 2011 ACCF/AHA/HRS focused updates incorporated into the ACC/AHA/ESC 2006 Guidelines for the management of patients with atrial fibrillation: a report of the American College of Cardiology Foundation/American Heart Association Task Force on Practice Guidelines developed in partnership with the European Society of Cardiology and in collaboration with the European Heart Rhythm Association and the Heart Rhythm Society. J Am Coll Cardiol 2011;57(11):e101-98.

2. Meinertz T, Kirch W, Rosin L, Pittrow D, Willich SN, Kirchhof $P$. Management of atrial fibrillation by primary care physicians in Germany: baseline results of the ATRIUM registry. Clin Res Cardiol 2011;100(10):897-905.

3. Price E, Baker R, Krause J, Keen C. Organisation of services for people with cardiovascular disorders in primary care: transfer to primary care or to specialist-generalist multidisciplinary teams? BMC Family Practice. 2014;15: 158.

4. Falces $C$, Andrea R, Heras M, Vehi C, Sorribes M, Sanchis $\mathrm{L}$, et al. [Integration Between Cardiology and Primary Care: Impact on Clinical Practice]. Rev Esp Cardiol 2011;64(7):564-71. Spanish.

5. Conti A, Canuti E, Mariannini Y, Viviani G, Poggioni C, Boni $\mathrm{V}$, et al. Clinical management of atrial fibrillation: early interventions, observation, and structured follow-up reduce hospitalizations. Am J Emerg Med 2012;30(9):1962-9.

6. Harrington AR, Armstrong EP, Nolan PE Jr, Malone DC. Cost-effectiveness of apixaban, dabigatran, rivaroxaban, and warfarin for stroke prevention in atrial fibrillation. Stroke 2013;44(6):1676-81.

7. January CT, Wann LS, Alpert JS, Calkins H, Cigarroa JE, Cleveland JC, et al. 2014 AHA/ACC/HRS guideline for the management of patients with atrial fibrillation: a report of the American College of Cardiology/American Heart Association Task Force on Practice Guidelines and the Heart Rhythm Society. J Am Coll Cardiol 2014;64(21):e1-76.

\section{Declaration of interests}

Nothing to declare.

\section{Acknowledgements}

Thomas M Munger MD, Peter A Smars MD, Merri L. Bremer RN RDCS, Lauren E Tarbell MS APRN CNS, Stephanie G Witwer MS Ph.D RN NEA.-BC, Jane F Myers APRN CNP, Shawna D Bellew MD, Susan K Graff. 
BMJ Quality Improvement Reports

\section{Ethical approval}

This quality improvement project was exempt from ethics review at Mayo Clinic Rochester (Minnesota, USA). 